tient, and these quickly crystallize into increased efficiency. ${ }^{1}$ When these patients were tested, the space over which powerful impressions were felt, while a light touch was not felt, formed a belt of pretty constant width, varying from two to three centimetres, outside the shaded areas, except in case No. 1, where it comprised the whole area between the dotted and the continuous lines.

Indeed, for case No. 1 we are compelled to conclude either that the circumflex was less severely damaged, or that its exclusive domain was more than usually contracted. Taking the partial anæsthesia as a sign of at least partial injury, however, the anatomical evidence afforded by this case corroborates strikingly that given by the others.

The methods employed in examining these cases were for the most part those which I ordinarily adopt, namely, the use of the wire brush, or, better, a single wire attached to the faradic battery, for the stronger impressions, and for the lighter a bit of string, guaged as to length, etc., by experiments on the healthy skin of each patient. . In the diagrams the shaded parts indicate the anæsthetic areas.

The absence in the drawings of the usual outline of the arm and shoulder is due to muscular atrophy, which was most noticeable in Case 3 , being partially masked in 1 and 2 by the presence of abundant subcutaneous connective tissue.

\title{
A CASE OF UNUSUAL INJURY FROM A CIRCULAR SAW
}

BY GEORGE JEWETT, M. D., FITCHBURG.

June 19, 1877, a young man was engaged in polishing a large circular saw, in rapid motion, when his clothes were caught by the teeth, and the anterior aspect of the right thigh was drawn upon the saw with the following results: The saw entered the thigh about six inches above the articulating surface of the knee-joint, cut off all the soft parts to the bone, the fascia lata, rectus, and triceps cruris being entirely severed. The anterior articulating surface of the external condyle of the femur was sliced off an inch and a half in diameter. This cut in the bone looked obliquely outward. At this period of the accident the joint having been entirely laid open, and the flap containing the patella having dropped down, the relation of the knee to the saw changed, and a vertical cut was made nearly parallel to the long axis of the femur, one inch from the first cut toward the median line. In this condition the wound was presented for treatment. The patient was thoroughly etherized, the ridge between the two bone cuts was removed, and the jagged anterior surface of the condyle smoothed off. To prevent accumulation of fluid in the synovial sac, a finger was passed into the most

1 Vide Létiévant, Sections nerveuses. 
depending part, and a bistoury pushed through externally near the head of the tibia. A new gum catheter filled with small holes was used for a drainage tube, and the joint well washed out with carbolized water.

The patella, like other parts in this region, had also been badly torn on its outer margin and articulating surface by the saw teeth. These fragments and inequalities were pared off, and the parts restored as far as possible to their normal position, and there retained by sutures. I now observed considerable loss of substance in the soft parts covering the external condyle. This gap was filled with carbolized lint, and the leg laid upon a straight ham splint : a carbolized compress enveloped the whole joint, and over all a roller was passed from the ankle to near the body, and the patient having suffered but little from loss of blood or shock was left in a comfortable condition. During the first four days the dressings were undisturbed, after which the compresses were removed, and but slight discharge was observed either from the cut surface or from the drainage tube. At no time did there seem to be fluid in the joint, and at the end of ten days the improvised drainage tube was removed permanently. The opening, however, did not heal for several weeks, but continued to wear the pouting everted lips peculiar to a fistulous opening.

The degree, duration, and locality of the pain suffered are worthy of remark. At no time was there much pain in the region of the wound, but for the first ten days it was mainly confined to the ankle and foot of the affected limb, and from this time to advanced convalescence it was almost entirely in the well knee, ankle, and foot, for the relief of which morphine was given somewhat freely. Convalescence was prolonged by two troublesome incidents: the granulations which filled the gap in the parts covering the external condyle were soft, flabby, and liable to bleed. Healthy skin was finally obtained after continued graduated compression, with occasional application of arg. nit. in stick. The other difficulty arose from the repeated absorption of portions of cicatricial tissue in the flap (which seemed to unite by first intention) apparently from lack of sufficient nourishment.

The thigh on the affected side is somewhat atrophied, and measures at the body more than two inches less than its fellow, fifteen months having elapsed since the accident. Although the leg cannot be perfectly flexed upon the thigh, and is still both smaller and weaker than its fellow, the patient now walks without limp, halt, or other evidence of the terrible wound from which he has thus far recovered. 